\section{Confederations and human ecology}

SIR-It was argued in Nature's Opinion columns ${ }^{1}$ that Europe has yet to get its act together as a genuinely integrated unit. And much is currently made of the expected efficacy of confederation. But does confederation represent natural progress or is it only a transient cultural fashion and are exhortations to throw off our xenophobic tendencies properly founded or should we forever be distrustful of unification? Ecology may provide some of the answers.

The $-3 / 2$ law of plant distribution ${ }^{2}$ has been shown to apply to animals as well as plants ${ }^{3}$. Not only can the natural relationship between mean size and distribution density of birds ${ }^{4}$, small mammals ${ }^{5}$ and primates in general ${ }^{6}$ be described by this theoretically substantiated ${ }^{7}$ allometric law but human distributions on the scale of nations also conform to it $^{8}$. What does it have to say about confederations?

The $-3 / 2$ law can be portrayed as:

$$
\log M=\log C-\frac{3}{2} \log \frac{N}{A}
$$

in which $M$ is the mean mass of a typical member of a community, $C$ is a constant relating to the energetics constraints of the species and physical environment, $N$ is the number of individuals and $A$ is the area of their habitat.

Therefore, from (1):

$\log M=\log C-\frac{3}{2} \log N+\frac{3}{2} \log A$ so $\log N M=\log C-\frac{1}{2} \log N+\frac{3}{2} \log A$ $=-\frac{1}{2} \log N+\left(\log C+\frac{3}{2} \log A\right)$

But $N M$ is the total mass of the community, say $M_{\mathrm{T}}$. Therefore, for a group occupying a fixed area so that $A$ is constant, we have from (2):

$\log M_{\mathrm{T}}=-\frac{1}{2} \log N+\log B$

where $B$ is the constant $C A^{3 / 2}$. Hence $M_{\mathrm{T}}=B N^{-1 / 2}$

Equation (3) describes a situation in which $M_{\mathrm{r}}$ increases as $N$ decreases in a given constant area of unaltering physical environment occupied by a group of biological entities subject to fixed energetics characteristics. This is the case for nations of humans subject to parallel cultural advances as is essentially the case in modern Europe". It is seen from equation (3) that, in order for the total mass (population) of a group of nations to expand, the number of independent nations must decrease. This implies the relinquishing of national boundaries to reduce wasteful competition in an ecological as well as economic sense.

The equivalent behaviour in plant communities involves the decay of one entity which imparts a resource to its neighbour in place of competition. In the case of international confederations the only decay that is required is of parochial xenophobia, the sacrifice of national identity to the more successful growth of the greater community. Alternatively, especially in the global village, individual nations can remain aloof and await the rewards of the next technological revolution in order to relieve their energetics constraints, in an ecological sense, and thereby continue to grow albeit at a rate inhibited by comparison with their more co-operative neighbours.

Generally speaking history appears to have eventually favoured the less-efficient but perhaps more individually satisfying second option. Whether history or ecology will have the greater influence on the future remains to be seen.

Bruce Denness

Bureau of Applied Sciences,

\section{Wydcombe Manor,}

Whitwell,

Isle of Wight PO382NY, UK

1. Nature 319, 249-250 (1986).

2. Yoda, K., Kira, T.J., Ogawa, H. \& Hozumi, K. J. Biol. Osaka) 14, 107 (1963)

3. Denness, B. Proc. int. Conf. Is the Earch a Living Organism? (National Audubon Society Expedition Institute, Sharon, Connecticut, 1986)

4. Schoener, T.W. Ecology, 49, 123-141 (1968).

5. McNab, B.K. Am. Nat. 97, 133-140 (1963).

. Clutton-Brock, T.H. \& Harvey, P. J. Zool. 183, 1-39 (1977)

Whittington, R. Nature 311, 217 (1984).

8. Hayton, A.F. Nature 310, 178 (1984).

\section{The mesoscale effects of nuclear winter}

SIR-The recent paper by Golding and colleagues $^{1}$ and accompanying News and Views commentary by Emanuel ${ }^{2}$ appear to represent an awakening of interest among mesoscale meteorologists in the problem of 'nuclear winter'. From Emanuel's characterization of the calculation of Golding et al. as "a welcome step in transforming nuclear winter research from a means of political advocacy to a scientific exercise", readers might well conclude that removal of nuclear war-generated aerosols by mesoscale processes had been ignored in previous studies, presumably rendering the results of these studies "unscientific". Further, it is rare that scientists of high calibre characterize the research of their colleagues - even controversial work - in terms as harsh as it "has become notorious for its lack of scientific integrity". We are moved to respond since we have published several contributions on "nuclear winter", including several refereed pieces in Nature ${ }^{3-7}$ and, in the process, have tried to refrain from political advocacy

The Golding et al. calculation is welcome since it is the only published mesoscale model applied to nuclear smoke that we know of, although it does not go nearly far enough to alter any possible conclusions connected with nuclear winter. For example, it has been well known and well stated in the major nuclear winter assessments $^{8,9}$ that early cloud-scale removal could be significant and was not explicitly included in any of the global circulation climatic models, whose grid scale resolution ranges from 500 to $1,000 \mathrm{~km}$. Indeed, the National Academy of Sciences assumed that some $50 \%$ of initially produced smoke would be washed out in cumulus scale convection, and cloud-scale calculations by William Cotton of Colorado State University ${ }^{10}$ have already shown that initial intense heating of the surface from urban mass fires is very likely to generate large-scale convective activity. None of this is ignored in the bulk of the nuclear winter literature that we are aware of, and it is spelled out explicitly in all of our papers.

For example, in our first paper we specifically state that "the way fires will burn (for example, fire storms), the height to which smoke is injected, the duration of fires, the particle concentration within initial smoke plumes, and early particle removal by rainout in convective/mesoscale circulations all occur on spatial scales smaller than the resolution of any general circulation model now available".

Moreover, in a civil exchange with S.F. Singer in the pages of Nature $e^{11,12}$, we conclude our discussion of Singer's criticisms with the following comment: "Mesoscale effects will be important in determining the removal of smoke from the atmosphere. Intuitively, one might expect enhanced moist convective activity around smoke clouds to increase the removal rate of particles, but it is not yet possible to explicitly caculate the effects of dense smoke clouds on mesoscale circulations."

Again, both in a semi-popular article and in the pages of Nature ${ }^{6}$, two of us have individually mentioned the uncertainties in applying the theory of nuclear winter on a local scale because of the mesoscale problem. Although the British Meterological Office calculation is a step in the right direction, it does not include the most controversial and important aspects in the entire early removal debate: how individual smoke particles from multiple smoke plumes will be processed as they pass through fire-associated cumulus clouds and then through secondary mesoscale circulations, and the fraction of the initially generated smoke that will not be processed by such convective activities. Indeed, this parameterization of microphysics is just as much a problem in cloud scale, mesoscale and general circulation models, and is, in our opinion, the principal uncertainty in determining whether early removal will prove to be a $90 \%$ or a $10 \%$ issue. The US National Academy Repor ${ }^{8}$ suggested early removal of $50 \%$ (on arbitrary grounds), and thus we still await further work.

In summary, we are content that our 